UCRL-JC-132481

Preprint

\title{
Material Characterization Using a Hyperspectral Infrared Imaging Spectrometer
}

\author{
Randy S. Roberts \\ William D. Aimonetti \\ Jay V. Bixler
}

This paper was prepared for submittal to

Institute for Electrical and Electronics Engineers Asilomar

Conference on Signals, Systems and Computers

Pacific Grove, CA

November 1-4, 1998

October 30, 1998

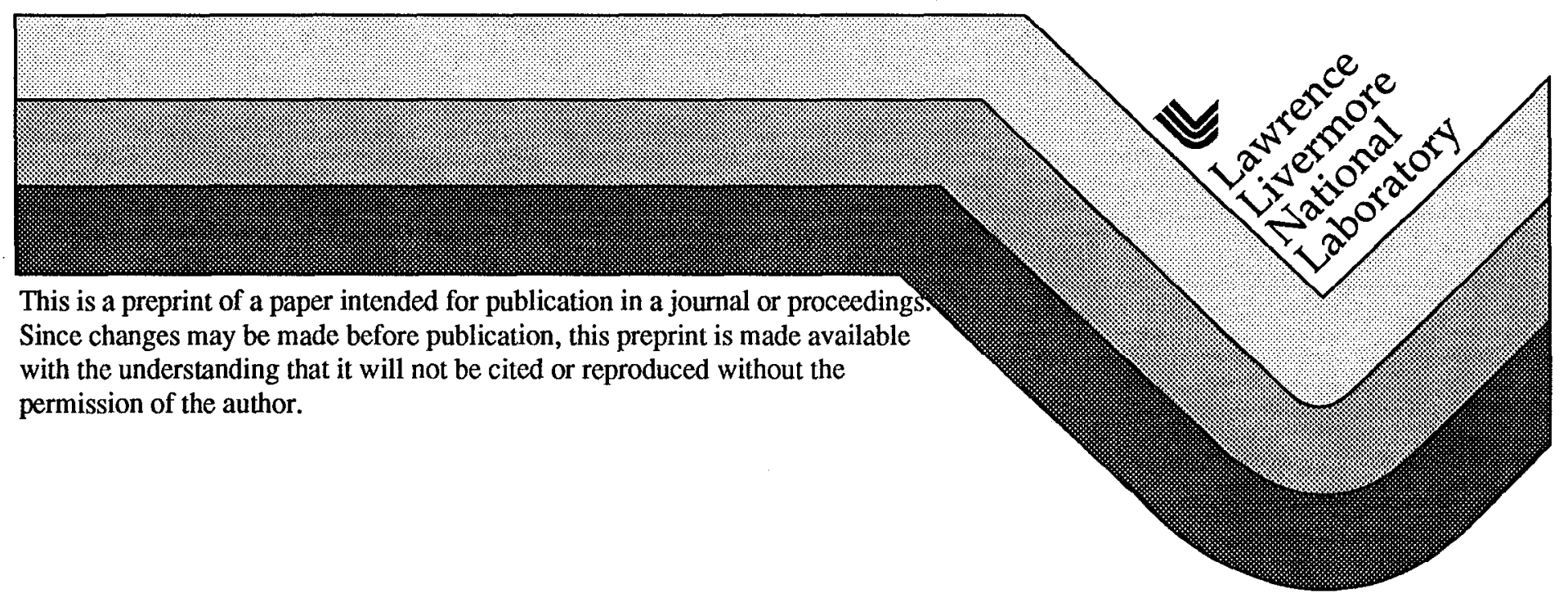




\section{DISCLAIMER}

This document was prepared as an account of work sponsored by an agency of the United States Government. Neither the United States Government nor the University of California nor any of their employees, makes any warranty, express or implied, or assumes any legal liability or responsibility for the accuracy, completeness, or usefulness of any information, apparatus, product, or process disclosed, or represents that its use would not infringe privately owned nights. Reference herein to any specific commercial product, process, or service by trade name, trademark, manufacturer, or otherwise, does not necessarily constitute or imply its endorsement, recommendation, or favoring by the Unitcd States Government or the University of California. The views and opinions of authors expressed herein do not necessarily state or reflect those of the United States Government or the University of California, and shall not be used for advertising or product endorsement purposes. 


\title{
Material Characterization Using a Hyperspectral Infrared Imaging Spectrometer
}

\author{
Randy S. Roberts, William D. Aimonetti, and Jay V. Bixler \\ Lawrence Livermore National Laboratory \\ Livermore, CA 94550 USA
}

\begin{abstract}
Fourier transform spectroscopy has found application in many areas including chemometrics, biomedical and biochemical studies, and atmospheric chemistry. This paper describes an investigation into the application of the LLNL Hyperspectral Infrared Imaging Spectrometer (HIRIS) to the non-destructive evaluation of man-made and naturnl materials. We begin by describing the HIRIS system and the objects studied in the investigation. Next, we describe the technique used to collest the hyperspectral imagery, and discuss the processing required to transform the data into usable form. We then describe a technique to analyze the data, and provide some preliminary results.
\end{abstract}

\section{Introduction}

Fourier Transform Infrared Spectroscopy (FTIS) has proven to be a valuable analysis tool in such arcas as chemometrics, biomedical and biochemical studies, and atmospheric chemistry [1-3]. One technique for performing FTIS is based on a Michelson interferometer [4]. Typically, a Michelson interferometer consists of two mirrors oriented along orthogonal axes. One of the mirrors is held in a fixed position, while the other mirror is allowed to translate along its axis. A beam splitter is placed between the mirrors such that light entering the interferometer is passed to each mirror. The light is reflected from the mirrors, combined by the beamsplitter, and focused onto a detector array. As the movable mirror in the interferometer translates, a time varying interference pattern is formed on the detector array. The time-series associated with each detector (pixel) in the array is called an interferogram. Fourier transformation

This project was supported by Engineering NDE Thrust Area funding. This work was performed under the auspices of the U. S. Department of Energy by Lawrence Livermore National Laboratory under Contract W-7405-Eng-48. of the interferogram reveals the spectral characteristics of the light input to the interferometer.

FTIS provides a fast, high resolution approach to the spectral analysis of broadband infrared light. Indeed, many molecules can be identified by the wavelengths of infrared light that they absorb or emit. In the case of absorption FTIS, a broadband source of photons is applied to a collection of molecules. The identities of the constituent molecules are found by determining which wavelengths are absorbed. This approach is often used to study the atmosphere or gaseous plumes where the thermal background is used as a wideband source of photons. In contrast, emission spectroscopy identifies the constituent components of an object by the wavelengths of photons emitted from a thermally excited object. Emission spectroscopy is employed when an object is (or can be) thermally excited. Emission FTIS is often used in material studies.

The Hyperspectral Infrared Imaging Spectrometer (HIRIS), developed at LLNL, was used to collect data for this investigation. (See reference [5] for a discussion of the predecessor to HIRIS.) While it has primarily been used in remote sensing applications, the focus here is to assess its usefulness in non-destructive evaluation applications. In essence, the HIRIS consists of a BioRad 896 Michelson interferometer, a cryogenically cooled Silicon Arsenide (Si:AsBIB) focal plane array, and associated optics. For these experiments, the HIRIS was used with a collimator lens to the input of the interferometer. The focal length of collimator lens was $100 \mathrm{~mm}$ and the focal length of the condenser lens was $226 \mathrm{~mm}$ thereby yielding a system magnification factor of 2.26 . The focal plane array in the HIRIS is a $128 \times 128$ pixel array with a pixel size of $75 \mu \mathrm{m}$ per pixel. The resulting resolution of the reconfigured system in thus on the order of $33 \mu \mathrm{m}$ per pixel.

Two objects were used to assess the applicability of this technology to non-destructive evaluation: a block of aluminum alloy A356 and a sample of granite rock. The 
A356 alloy consists primarily of aluminum ( $92 \%)$, silicon $(\sim 7 \%)$ and trace amounts of copper, magnesium, manganese, iron and zinc [6]. This alloy has excellent corrosion resistance and casting properties, good machinability and weldability, and high strength. It is most often used in the automotive and aircraft industries where high strength and low corrosion are required. The block we analyzed was subject to compression-tension cycles until it fractured. Our interest in this sample is to study the fracture sites. Our second object of interest was a sample of granite rock. Granite is an igneous rock typically consisting of quartz, feldspar and some ferromagnesian minerals such as mica, amphibole or py, $0 x-$ ene [7]. Chemically, granite is composed of $\sim 70 \%$ silica $\left(\mathrm{SiO}_{2}\right), \sim 15 \%$ Aluminum Oxide $\left(\mathrm{Al}_{2} \mathrm{O}_{3}\right)$ and trace amounts of other oxides. Our interest here is to study the mineral and chemical content of the sample.

\section{Data Collection And Processing}

The A356 block and granite rock were heated with a hot air gun until they reached a temperature of approximately $45^{\circ} \mathrm{C}$. The samples were held at this temperature for the duration of the data collection. For these experiments, the spectral resolution of the interferometer was set to $8 \mathrm{~cm}^{-1}$. (Note that frequency is measured in wavenumbers with units of reciprocal centimeters, i.e., the number of wavelengths contained in one centimeter.) In all, 711 frames of $128 \times 128$ images were produced for each scan. As a point of terminology, the sequence of images produced by a scan is referred to as a raw data cube. The interferogram associated with pixel $(m . n)$ in the $i^{\text {in }}$ raw data cube is denoted as $x_{i}(m, n, \tau)$ where $\tau$ is the time index $0 \leq \tau \leq 711$.

In order to increase the signal-to-noise ratio of spectral features, an ensemble of 16 raw data cubes were collected from each sample. These cubes were averaged into a single data cube:

$$
x(m, n, \tau)=\left\langle x_{i}(m, n, \tau)\right\rangle_{i}
$$

In addition to data from the $A 356$ block and granite rock, calibration data was collected from a black body source. The source was set to $T_{H}=55^{\circ} \mathrm{C}$ for a hot reference, and $T_{C}=40^{\circ} \mathrm{C}$ for a cold reference. For each sample, 16 raw data cubes were collected of the hot and cold references. These data cubes were averaged in the manner previously indicated. As a result of the averaging, three data cubes were produced for each experiment: a sample data cube $x(m, n, \tau)$, a hot black body data cube $h(m, n, \tau)$, and a cold black body data cube $c(m, n, \tau)$.

An exemplar interferogram from the A356 experiment is shown in Figure 1. This interferograra is from

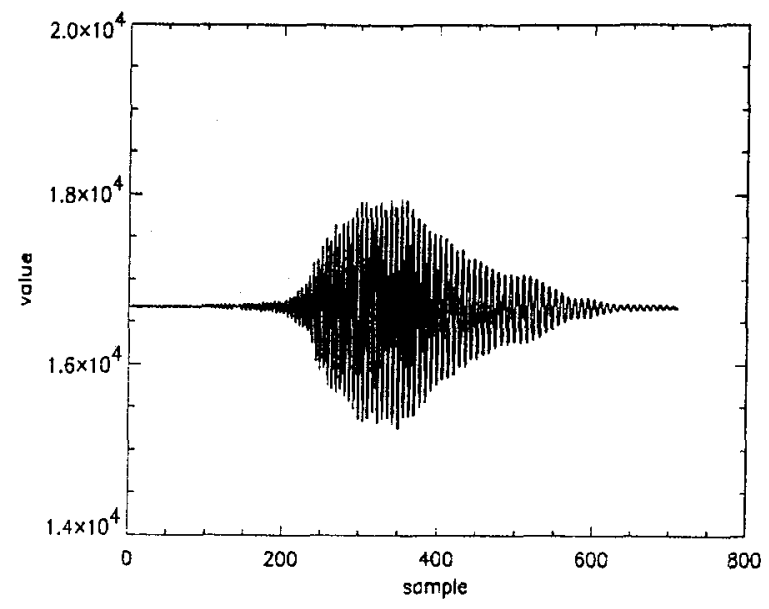

Figure 1: Interferogram from pixel $(64,64)$ of the A356 sample. The centerburst is located between samples 200 and 600 .

pixel $(64,64)$ of the sample. Note that the interferogram appears as a modulated sinusoid. The region between the $200^{\text {th }}$ and $600^{\text {th }}$ sample is called the centerburst of the interferogram. An image associated with the centerburst portion of a data cube, say for example $x(\cdot, \cdot, 400)$, appears as a pattern of concentric interference rings. Interferograms often exhibit low amplitude signals on either side of, and perhaps within, the centerburst. These signals are a result of channel echo, a reflection of energy back into the interferometer from the focal plane array. The interferogram illustrated in Figure 1 does not exhibit channel echo effects due to the coarse spectral resolution used in these experiments. Interferograms, while illuminating some signal structure in a data cube, are difficult to interpret. However, the spectral information contained in interferograms can be quite revealing.

The spectral content of a data cube is found by Fourier transforming each interferogram in the data cube. Typically, a data-tapering window $w(\tau)$ is applied to each interferogram to reduce channel echo. To improve computational efficiency, the interferograms are often zero padded to an appropriate length. The magnitude of the resulting spectral data cube is given by

$$
\bar{X}(\cdot, \cdot, k)=|F\{w(\tau) \tilde{x}(\cdot, \cdot, \tau)\}|
$$

where $\tilde{x}$ is the zero-padded interferogram. In these experiments, a data-tapering window was not applied due to the apparent lack of channel echo. Each interferogram was zero padded from 711 samples to 1024 samples. Similar processing yields $H(\cdot, \cdot, k)$ and $C(\cdot, \cdot, k)$, the hot and cold black body reference data cubes. Finally, the out-of-band portions of the spectra 


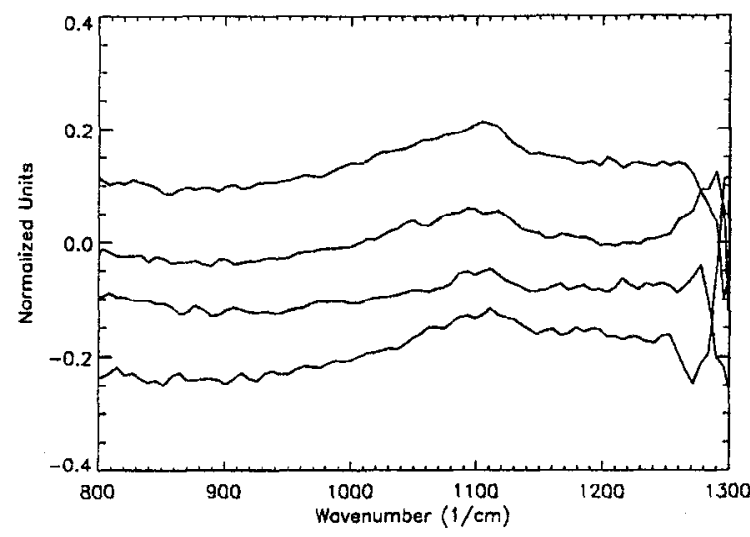

Figure 2: Spectrum plot of the A356 sample in normalized units. From the top, the spectra are from pixels $(84,22),(78,34),(69,65)$, and $(26,22)$.

were discarded. Only 100 samples in the band $700 \mathrm{~cm}^{-1}$ to $1310 \mathrm{~cm}^{-1}$ were kept.

After the spectral data has been averaged, windowed, zero-padded and fourier transformed, sample data is calibrated using the hot and cold references. Calibration is performed using the following expression

$$
X(m, n, k)=\frac{\tilde{X}(m, n, k)-C(m, n, k)}{H(m, n, k)-C(m, n, k)}
$$

where the units are arbitrary. To convert the calibrated data cube into spectral radiance, the following relationship is used:

$$
S(m, n, k)=X(m, n, k) \Delta B(k)+B(k, T)
$$

where $B(k, T)$ is the Planck spectral radiance function [3], [4]

$$
B(k, T)=\frac{2 h c^{2} k^{3}}{e^{h c k / K T}-1}
$$

and

$$
\Delta B(k)=B\left(k, T_{H}\right)-B\left(k, T_{C}\right)
$$

In the Plank spectral radiance equation, $k$ is the wavenumber of the radiation, in units of $\mathrm{cm}^{-1}, T$ is the temperature of the cold black body in degrees Kelvin, $h$ is Plank's constant, $c$ is the speed of light, and $K$ is Boltzmann's constant.

\section{Data Analysis}

Spectra in a calibrated data cube can reveal a great deal of information about the object under study. In a very pure sample, it might be possible to identify the material by comparing its spectrum to the spectra in a materials library. Libraries of material spectra are avail-

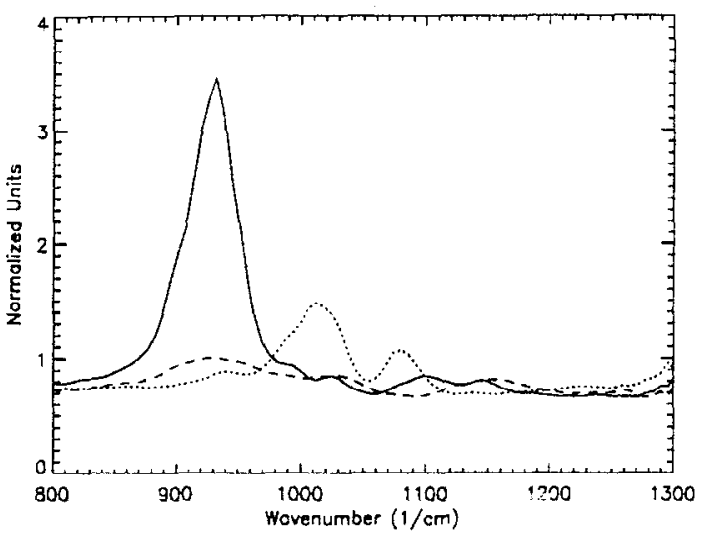

Figure 3: Spectrum plot of the granite sample in normalized units. Solid line: pixel $(48,52)$; Dotted line: pixel $(91,37)$; Dashed line: pixel $(87,75)$.

able on the world wide web at various locations (see for example [8]). As examples, Figures 2 and 3 illustrate several spectra from the A356 and granite samples. The A356 spectra in Figure 3 are from pixels within a $1.9 \mathrm{~mm}$ by $1.4 \mathrm{~mm}$ area. They have a very flat response over the $800 \mathrm{~cm}^{-1}$ to $1300 \mathrm{~cm}^{-1}$ spectral band. Inspection of the A356 sample reveals a homogeneous surface, one that tends to support the flat response seen in Figure 2. Moreover, spectra for sheet aluminum have a similar flat response in this band [8]. Observe the negative spectrum values in Figure 2. These values are a result of calibrating the data cube with a cold black body source that is warmer than the sample.

In contrast, spectra from the granite sample reveal a great deal of structure. The spectra in Figure 3 were collected from an area of $1.4 \mathrm{~mm}$ by $1.25 \mathrm{~mm}$. As seen in the figure, the spectrum from pixel $(48,52)$ has a large response centered around $930 \mathrm{~cm}^{-1}$. The spectrum of one type of granite has a similar response in this same spectral band [8]. The spectrum associated with pixel $(91,37)$ has two smaller peaks centered around $1020 \mathrm{~cm}^{-1}$ and $1080 \mathrm{~cm}^{-1}$. The type of mineral associated with this spectrum has not been identified. The remaining spectrum exhibits little structure across the band.

In many cases the spectrum associated with one pixel in a calibrated data cube is a complex mixture of many different material spectra. It is therefore highly desirable to transform the spectra into a form that is more readily analyzed. One approach that is well suited for analyzing such random data is the Karhunen-Loeve Transformation (KLT), also known as the method of principle components [9], [10], [11]. 


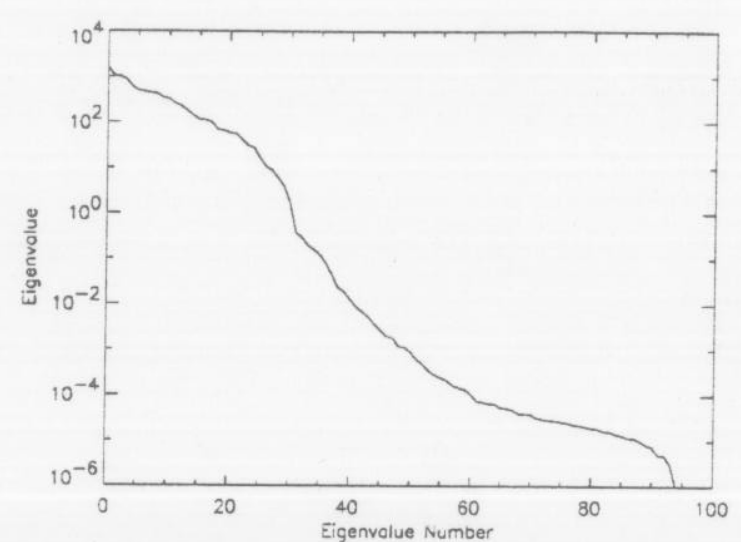

Figure 4: Plot of the spread of eigenvalues in the granite sample.

To apply this technique to the analysis of a calibrated data cube, we model the spectra in the data cube as an ensemble of random vectors. The ensemble is formed by mapping the data cube $X(m, n, k)$ into the matrix $\boldsymbol{X}(k, p)$ where the $k^{\text {th }}$ spectral image is mapped into the $k^{t h}$ row of $\boldsymbol{X}$. Denote the number of rows in $\boldsymbol{X}$ as $n_{k}$, and the number of columns as $n_{p}$. As the KLT relies upon second order statistics of the data, it is necessary to estimate the covariance matrix of the spectra. Begin by removing the bias on each row in $\boldsymbol{X}$ by subtracting the average value of the row from each element in the row:

$$
\overline{\boldsymbol{X}}(k, \cdot)=\boldsymbol{X}(k, \cdot)-\langle\boldsymbol{X}(k, \cdot)\rangle
$$

The covariance matrix is then estimated as

$$
C=\bar{X} \bar{X}^{T}
$$

where the resulting covariance matrix has dimensions $n_{k} \times n_{k}$. Next, the eigenvalues $\lambda_{i}$ and associated eigenvectors $\phi_{i}, 1 \leq i \leq n_{k}$, of the covariance matrix are computed. The eigenvalues are ordered from the largest to smallest,

$$
\lambda_{1} \geq \lambda_{2} \geq \lambda_{3} \geq \ldots \geq \lambda_{n_{k}}
$$

and their associated eigenvectors arranged into an eigenvector matrix $\Phi$

$$
\Phi=\left(\phi_{1}, \phi_{2}, \ldots, \phi_{n_{k}}\right)
$$

A member of the spectral ensemble, say $x$, can be represented as a weighted sum of the eigenvectors $\boldsymbol{x}=\Phi \boldsymbol{y}$, where $\boldsymbol{y}$ is a vector of weights. Conversely, in the inverse relation $y=\Phi^{H} x$, where the superscript represents the Hermitian transpose, $y$ is the KLT of $x$.

As a result of the basis restriction properties of the $\mathrm{KLT}$, the first few eigenvectors represent the most important features in the data [10]. It is instructive to examine the ordered eigenvalue sequence. A plot of the

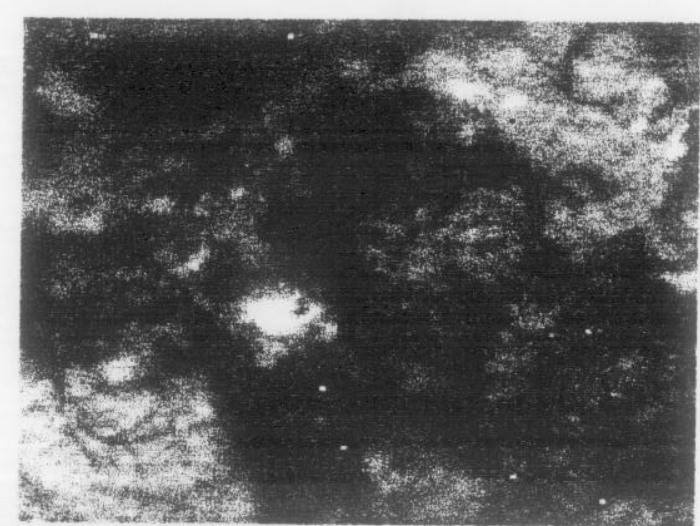

Figure 5: Eigenimage of the granite sample. This eigenimage covers a $4 \mathrm{~mm}$ by $4 \mathrm{~mm}$ region on the rock

eigenvalues of the granite sample is shown in Figure 4. As illustrated in the figure, the magnitude of the eigenvalues decreases rapidly over the first twenty or so modes. These modes comprise the main structure of the data. If the data were reconstructed from these modes, the residual error would be minimal. From approximately the twentieth fifth to the ninety-fourth eigenvalue, the decrease is more gentle and the eigenvalues have a small magnitude. These modes are associated system noise and artifacts, and are not important to the material analysis. The remaining eigenvalues belong to degenerate modes. They are produced by the zero padding applied to the interferograms before Fourier transformation, and are not useful in the analysis [5].

The spatial structure of a data cube decomposed with the KLT is revealed in a series of eigenimages. The $i^{t h}$ eigenimage is the projection of the spectra in the data cube onto eigenvector $\phi_{i}$. An example eigenimage from the granite rock is shown in Figure 5. This eigenimage covers an approximately $4 \mathrm{~mm}$ by $4 \mathrm{~mm}$ area of the rock, and exhibits the complex spatial structure characteristic of granite. The various regions in the image correspond to the constituent components of granite, namely, quartz, feldspar and mica. The mineral identity of the various regions in the eigenimage has not been established at this time, but is under study. Note that there are several black and white single-pixel features in the image. These features are bad pixels in the focal plane array, and are not of concern in the analysis.

\section{Summary}

This investigation assessed the use of HIRIS in the nondestructive evaluation of man-made and natural objects. Hyperspectral infrared data was collected on 
two objects, a block of aluminum alloy and a specimen of granite rock, after a slight modification of the HIRIS input optics. Several data sets were collected on the specimens and calibration sources. The data sets were averaged to increase the signal-to-noise ratio, processed and Fourier transformed to produce data cubes of calibrated spectral data. Preliminary analysis of the spectral data using KLT techniques reveals interesting structure in the data, but additional study is required to fully assess the potential of this technology.

\section{Acknowledgments}

The authors would like to thank Ken Dolan for providing A356 samples, Brett Wayne for his assistance in the data collection, Bob Priest and Charlie Bennett for reviewing the manuscript, and Harry Martz and Mike Carter for their interest and support of this project.

\section{References}

[1] Q. Ding and G. Small, "Wavelength Selection for the Determination of Glucose in Human Serum by Near-Infrared Spectroscopy," Fourier Transform Spectroscopy: 11 th Intemational Conference, edited by J. A. de Haseth, American Institute of Physics, 1998.

[2] D. Byler, et. al., "Effect of Sucrose on the Thermal Denaturation of a Protein: An FTIR Spectroscopic Study of a Monooclonal Antibody," Fourier Transform Spectroscopy: 11th International Conference, edited by J. A. de Haseth. American Institute of Physics, 1998.

[3] W. Taub, "Atmospheric FTS," Fourier Transform Spectroscopy: 11th International Conference, edited by J. A. de Haseth, American Institute of Physics, 1998.

[4] R. Beer, Remote Sensing by Fourier Transform Spectrometry, Wiley, 1992.

[5] C. L. Bennett, "LIFTERS, The Livermore Imaging FTIR Spectrometer," Fourier Transform Spectroscopy: 11 th Intermational Conference, edited by J. A. de Haseth, American Institute of Physics, 1998.

[6] ASTM Metals Reference Book, 2nd edition.

[7] C. E. Tilley, "Granite," Encyclopedia Britannica, 1959 edition.

[8] Jet Propulsion Laboratory web site http://speclib.jpl.nasa.gov

[9] E. Malinowski, Factor Analysis in Chemistry, 2nd edition, Wiley, 1991.

[10] K. Fukunaga, Introduction to Statistical Pattem Recognition, Academic Press, 1990.

[11] A. K. Jain, Fundamentals of Digital Image Processing, Prentice Hall, 1989. 